\title{
LA REFORMA CULTURAL EN CHILE: UN EJEMPLO DE POLARIZACIÓN EN LA GESTIÓN DE UNA POLÍTICA PÚBLICA
}

\author{
Norma Muñoz Del Campo (normamdelc@gmail.com) \\ Universidad del Pacifico, Chile
}

El artículo busca explicar las diversas dinámicas y problemáticas que condujeron a la creación del Consejo Nacional de la Cultura y las Artes en Chile. Al utilizar como caso de estudio la creación de una institución pública, el trabajo analiza las variaciones que el proyecto sufre durante su puesta en marcha. Se argumenta que la institucionalidad pública actual es resultado de una permanente tensión entre dos paradigmas de ejecución de políticas públicas (el cultural y el referencial global).

Palabras clave: análisis cognitivo; politicas públicas; Tres I; politica cultural.

\section{CULTURAL REFORM IN CHILE: AN EXAMPLE OF POLARIZATION IN PUBLIC POLICY MANAGEMENT}

This article seeks to explain the different dynamics and issues which led to the creation of the Chilean National Council for Arts and Culture. Using the creation of a new public institution as a case study, the paper seeks to analyze the changes the project experienced during its initial stages. It is argued that public institutions currently suffer from a permanent tension between two different paradigms in the execution of public policy (cultural and global).

Keywords: cognitive analysis; public policy; cultural policy. 


\section{INTRODUCCIÓN}

Emprender una reflexión sobre la relación estado-cultura constituye un trabajo en continua reconstrucción dado el carácter impreciso del término "cultura". Se trata de un concepto polémico que despierta sentidos e interpretaciones diversas y que como tal ha creado continuamente espacios de encuentro y de disputa. En este sentido destacan los estudios realizados principalmente en Francia donde se encuentran una serie de investigaciones sobre la relación entre el estado y la cultura desde el marco analítico de las políticas públicas (Dubois, 1999; Sáez, 1985; Surel, 1997; Urfalino, 2004).

La literatura dedicada a esta particular relación pone en evidencia elementos característicos de la acción del estado. El primero está relacionado a la ambigüedad que rodea y construye el concepto "cultura", ya que analizar la política cultural implica estudiar "la imagen que los poderes públicos que impulsan políticas en la materia tienen de la cultura” (Poirrier, 1998), pero también implica considerar "la idea" que esos mismos poderes públicos se hacen de su rol en ese campo. La incertidumbre primordial que está al origen de esta intervención introduce un segundo elemento que es la legitimidad de acción: ¿Qué es lo que puede -o no puede- hacer el estado en materia cultural? ¿Cuáles son los límites de su intervención? Se trata de preguntas que, como veremos, atraviesan los estudios de política pública y más precisamente de política cultural. Finalmente, el tercer elemento característico de la acción pública en la materia está relacionado con la fragmentación institucional y a los recursos relativamente limitados destinados a las instituciones públicas que tienen responsabilidades (en este ámbito en comparación a los presupuestos de otros ministerios). Guy Sáez propone un estudio sobre la función que el estado debe asumir en materia cultural considerando que "toda política pública pone un problema de finalidad" y ésta implica que "un acuerdo estable y durable surge para reconocer la necesidad de intervención y de toma de responsabilidades del estado en la organización de estos ámbitos" (Sáez, 1985:387). Este autor destaca la dificultad que representa este sector porque "ni los responsables políticos, ni los investigadores se aventuran o arriesgan a definir claramente qué implica la acción pública en este ámbito" (Sáez, 1985:387). Dentro de esta misma perspectiva Vincent Dubois destacará que es la definición del "objeto mismo de investigación" la que varía enormemente de un país al otro 
y que la fragilidad institucional del sector genera "no un cuerpo de agentes del estado sino una multitud de oficios y de formaciones más o menos institucionalizadas" (Dubois, 1999:10).

Dubois propone que la política cultural resulta de "la dinámica interna de las instituciones, de reivindicaciones asociativas o políticas" y al mismo tiempo es el fruto del accionar de los actores que van a tener que "vencer las reticencias, los pensamientos instalados [...] operar una cierta modificación del estatuto del arte y la cultura en la vida social para entregar una legitimidad a la acción pública y así orientarla" (Dubois, 1999: 396). Estas luchas de legitimidad van a modelar un cuadro de acción pública que determinará la institucionalización de la cultura en los diferentes países.

La constitución de estas instituciones culturales ha estado marcada por dos modelos que se han transformado en referentes a nivel mundial: Por un lado la figura ministerial, el estado interventor, desarrollada en Francia, donde el estado tiene las mayores responsabilidades en el desarrollo del sector. Por otro lado encontramos el modelo de los Estados Unidos, compuesto de grandes instituciones culturales y patrimoniales autónomas y privadas. Estos dos modelos tipo representan, según Sáez (1985:395), "dos proyectos ideológicos distintos pero presentes en todos los sistemas implementados".

En torno a esta cuestión de legitimación aparece apropiado citar las declaraciones de Maryvone de Saint-Pulgent, quien fuera Consejera de Estado en Francia y Directora de Patrimonio en el Ministerio de la Cultura entre 1993-1995; ella hablará en una entrevista sobre la crisis existente al interior de esta relación entre el estado y la cultura:

¿Qué puede hoy fundar la acción cultural pública? Sería por ejemplo como lo pensaba Malraux, luchar contra los efectos del mercado (...) donde la legitimidad del estado está en preservar la gran cultura, los grandes maestros del arte contra la invasión del mercado. Esta proposición doctrinaria está hoy completamente sobrepasada por la idea mucho más moderna que consiste en decir que es imposible jerarquizar entre las diferentes formas de cultura (...) el rol del estado es de proteger la diversidad y la libertad de expresión. En este momento 
el problema es que el estado llega tanto a respaldar las expresiones culturales que ya cuentan con el apoyo del mercado como las otras, y cuando hay que elegir, ¿cuál elegir? ¿La del mercado porque es la de la mayoría o la del "no mercado" porque es aquella que hay que proteger? El estado no logra decidir y sobre todo no logra argumentar su decisión. Hay una crisis ideológica mayor en la cultura, que es igualmente una crisis política (Calmettes y Djian, 2003).

Es posible observar cómo esta relación se funda sobre una legitimidad política cuya construcción es compleja, aún más con el desarrollo de sociedades modernas y la importancia atribuida al mercado, que produce la aparición de nuevas dinámicas a veces contradictorias.

Junto con abordar el tema de la legitimidad es necesario contextualizar el concepto de política cultural. Antes que todo, es necesario destacar que referirse a este concepto se traduce en hacer una reflexión tácita sobre la sociedad que se desea construir y que dada la complejidad de este concepto se destacarán aquí dos puntos principales que permitirán contar con dos elementos de análisis que contribuirán al estudio propuesto en este artículo.

El primero es que los diferentes estudios sobre política cultural destacan que la intervención del estado en la materia ha existido siempre, sin embargo no es posible decir lo mismo de la "organización del ámbito a través de una política pública” (Sáez, 1985:390). Es decir, la intervención de diferentes gobiernos en las artes y la influencia que en esta materia tienen las administraciones públicas no son algo nuevo; al contrario, se trata de acciones antiguas, sin embargo constatar su existencia no representa en consecuencia la existencia de una política cultural (Dubois, 1999).

En segundo lugar es necesario subrayar lo propuesto por Philippe Urfalino en su obra La invention de la politique culturelle, donde se reconstituye la historia de la institución cultural francesa, mostrando cómo la política cultural nace en ese país en el año 1959 con André Malraux. Este autor muestra cómo la institucionalización de la cultura es el fruto de un proceso de sedimentación, no solamente sobre la base de adiciones o evoluciones político-administrativas, sino que sobre la base de ideas y concepciones que determinarán también las relaciones entre estado y 
cultura. Este sector de intervención pública nace, para el autor, estrechamente ligado al nacimiento de un proyecto que buscaba defender una causa más global, y en este sentido él distinguirá la política cultural de las políticas culturales. La primera reservada a "Alcanzar el alineamiento, es decir la aceptación, de una representación del rol que el estado desea que el arte desempeñe para cambiar o consolidar la sociedad a través de un conjunto de medidas públicas (nominaciones, financiamientos, creación de diversos dispositivos y establecimientos)" mientras que la segunda se refiere al "conjunto de medidas, o como se dice ahora, de políticas públicas de la cultura” (Urfalino, 2004:385).

En Chile, con la creación del Consejo Nacional de la Cultura y las Artes (CNCA), por primera vez en su historia el país contará con una institución superior en materia cultural. El interés de crear esta instancia había sido ya señalado de manera explícita en el programa presidencial del primer gobierno democrático de la post dictadura en 1990. A través de la creación de esta institución se funda en los albores del siglo XXI lo que se llamó en Chile, una política cultural de estado.

En virtud de lo expuesto, el presente artículo tiene como su preocupación central y se propone demostrar que la institucionalidad pública cultural es centro de luchas o tensiones entre distintos actores y campos de fuerzas, para ello se desarrolla una categoría de acción pública anclada en el análisis cognitivo de las políticas públicas (Jobert y Muller, 1987). Del mismo modo, se analiza la implementación y funcionamiento del CNCA, para ello se utiliza perspectiva de análisis centrado en las "Tres I" (Palier y Surel, 2005).

\section{UN ESTUDIO INSCRITO EN EL ANÁLISIS DE LAS POLÍTICAS PÚBLICAS}

Al mirar la cultura como política pública se hace necesario proponer una interrogante de base: ¿qué es una política pública? Muller y Surel subrayan el carácter polisémico del término "política" para definirlo a través de los vocablos ingleses polity, politics y policies, que evidencian sus diferentes dimensiones. El primero designa según estos autores la esfera política, marcando una diferencia entre el mundo de la política y la sociedad civil, el segundo se relaciona a la actividad política en general (la competición para la obtención de puestos políticos, el debate 
partisano, las diversas formas de movilización) y el tercero a la acción pública, es decir al "proceso a través del cual se elaboran e implementan los programas de acción pública" (1998:13). Las definiciones propuestas para delimitar este concepto consideran entonces dichas dimensiones. Una política pública es así "el resultado de la actividad de una autoridad investida de poder público y de legitimidad gubernamental" (Meny \& Thoening, 1992:89).

Ahora bien, la constante incertidumbre que caracteriza el contexto político, económico y social dentro del cual evoluciona el estado hoy, marcado por una multiplicidad de actores y de niveles de acción, hace pensar que no es únicamente la autoridad pública la que interviene en la definición de las acciones que se llevarán a cabo. Existen en efecto diferentes maneras de abordar las políticas públicas, por un lado "la manera clásica, donde las autoridades públicas intervienen según una "finalidad predefinida", a partir de objetivos y de medios definidos a priori que se dirigen a los receptivos" (Massardier, 2003:1), y por el otro a "la manera renovada, donde las autoridades públicas no poseen más el monopolio de fabricación de políticas públicas y deben "hacer con" una multiplicidad de actores que proyectan sus "finalidades vividas" en el proceso de políticas públicas". Así, frente a los enfoques que destacan la autoridad pública y la racionalidad del estado, surgen otras corrientes que permiten realizar un análisis más horizontal, integrando diferentes herramientas capaces de estudiar y de analizar nuevas realidades.

Es así que Lascoumes y Le Galès (2007) afirman que "el modelo clásico de las políticas públicas conducidas a título principal por un estado centralizado sobre sectores bien delimitados está sobrepasado" (pág. 5). Ellos exponen que hoy se está frente a múltiples niveles de acción espacial hacia arriba y abajo, al mismo tiempo que frente a "una proliferación de fuentes de influencia sobrepasando el marco nacional, a la introducción de mecanismos de mercado en las políticas públicas, a privatizaciones, a asociaciones publico-privadas y a métodos de gestión de la empresa” (Lascoumes y Le Galès, 2007:6), todo esto como producto del fenómeno de la globalización.

Tras el necesario posicionamiento dentro de estos debates, este estudio retiene la definición de política pública como "una actividad colectiva que participa en la creación de un orden social y político, en la dirección de la sociedad, en la regulación de sus tensiones, en la integración de grupos y a la resolución de conflictos" (Lascoumes y 
Le Galès, 2007:5). Esta orientación es coherente con el contexto de democratización, que acompaña el proceso estudiado, donde la reconstrucción del orden político, social y cultural era una prioridad. Se considera también el enfoque según el cual una política pública no es un programa estricto y racional sino más bien "una experimentación a observar en la implementación” (Lascoumes y Le Galès, 2007:42), un puzzle que pretende comprender cómo sus piezas se componen. Si se considera en efecto los vocablos polity, politics, policies para definir una política pública se hace necesario entonces comprender su articulación para trazar cómo una política emerge, qué implementación provoca y cuáles son los nuevos desafíos que redefine.

Una política pública representa, entonces, el encuentro entre objetivos (que pretende cumplir), actores (administrativos, los que dirigen la implementación, pero también y por sobre todo los que garantizan su funcionamiento en el día a día, en este caso los artistas, las asociaciones y fundaciones artísticas, los representantes regionales y locales), instituciones que constituyen el marco evolutivo de las interacciones (la adaptación -o no adaptación- de los actores a este marco), representaciones (que dan sentido a las diferentes acciones emprendidas), y finalmente un proceso (a través del cual la política va a "vivir", construirse y cuyo estudio permite comprender lo que está en juego y los resultados de la acción pública).

\subsection{El aporte del análisis cognitivo de políticas públicas inscrito en un análisis integrado de las "Tres i"}

A la luz de estas reflexiones, aparece entonces apropiado entender desde una perspectiva cognitiva de las políticas públicas, cómo se reflejarán estos elementos en el proceso de institucionalización de la cultura y cómo condicionan la vivencia misma de las políticas públicas culturales implementadas por el fruto de este proceso institucional, el CNCA. Se propone integrar el análisis cognitivo a una mirada tridimensional inspirada por el enfoque de las "Tres i", un concepto que designa "una serie de elementos de análisis de políticas públicas: las "ideas", los "intereses" y "las instituciones"” (Palier y Surel, 2005:10). Esto permite, en el caso del proceso de institucionalización de la cultura en Chile, proponer un análisis basado "en la idea de que estas variables explicativas no son exclusivas las unas de otras, pero pueden estar asociadas al menos a priori para delimitar los ejes de 
la investigación y las dinámicas pertinentes en el análisis del estado en acción” (Palier y Surel, 2005:10).

El análisis cognitivo posiciona una entrada al estudio de las políticas públicas que permite comprender la dimensión de las ideas y su influencia en los procesos de política pública. Este análisis surge en Francia en la década de los ochenta, asumiendo las políticas públicas como un "proceso de construcción de una relación al mundo" (Muller, 1995: 153). Esta lógica se basa en dos conceptos: Mediación y Referencial.

La lógica Referencial de una política pública se define como "la representación que uno se hace de un sector particular, así como también de su lugar y su rol en la sociedad" (Jobert y Muller, 1987: 65); se trata de un concepto que comporta tres elementos: el referencial global, el referencial sectorial y los elementos que marcan la integración del sectorial en el global. El Referencial global se relaciona a los marcos cognitivos y normativos en el centro de la acción pública y es definido como una imagen social de toda la sociedad, una representación global a la cual vendrán a inscribirse las diferentes representaciones sectoriales que sufrirán a su vez de un proceso de jerarquización. El referencial global podría simbolizar, por ejemplo, procesos políticos, sociales y/o económicos que enmarcan evoluciones sectoriales particulares. En este caso corresponde al proceso de democratización y de modernización y reforma del estado donde se inserta la reforma cultural. Por su parte, el referencial sectorial representa la imagen social de un sector particular y será conformado por las ideas propias a dicho sector y por los actores que participan en él. Estas percepciones no son siempre racionales ya que generalmente son el fruto de relaciones de fuerza entre los participantes de un sector dado.

El concepto de Mediación corresponde a un proceso que se promueve a través de "dos duplas", cada una de las cuales constituye una dimensión en sí misma. La primera dupla es la "cognitiva/normativa" relacionada con la "producción de sentido" (dimensión de las ideas), y la segunda pertenece al campo "intelectual/ poder" y en ese estatus está vinculada a la estructuración de un campo de fuerza y como tal relacionada a la dimensión de los actores. Es así como la noción de mediación permite aquí analizar un proceso que considera la constitución de una visión particular del mundo por un grupo social, que le permite posicionarse a sí mismo, así como también respecto a otros grupos que habitan el mismo "universo 
de sentido" y de esta manera comprender la construcción de un espacio de acción pública que implica una interacción entre el estado, la sociedad y el mercado.

Para completar la dimensión de las ideas se propone la noción de Paradigma presentada por Kuhn (2008) y aplicada al análisis de las políticas públicas (Surel, 1995). Es una noción coherente con el enfoque aquí presentado, ya que considera también las visiones del mundo y además presenta un sistema organizado por cuatro elementos cuya presencia e identificación marcan la constitución de un paradigma. Se trata de un marco disciplinario que permite identificar y estudiar modelos de acción pública, así como también comprender sus procesos de cambio.

La tercera dimensión que circunscribe este trabajo es la institucional. Considerar esta dimensión permitirá identificar y analizar los recursos institucionales en juego, así como también trazar las continuidades y cambios relativos a esta reforma. Las instituciones son definidas como las reglas, sean éstas formales o informales, como límites puestos por el hombre que orientan la manera como los individuos actúan en la vida cotidiana (North, 1990; Boussaguet, 2004:138).

Se distingue aquí la perspectiva propuesta por la literatura neo-institucionalista ya que pone el énfasis en explicar "cómo las instituciones nacen o se modifican" al mismo tiempo que busca comprender "cómo se construye la relación entre institución y comportamiento" (Hall y Taylor, 1997:469). Este enfoque considera que las instituciones "no son solamente creadas como un "espejo" de la sociedad o el simple producto de lógicas individuales, tampoco son un factor de orden exógeno al juego de actores, de la selección de líderes, y de la distribución de recursos. Ellas forman, al contrario, el marco evolutivo de estas interacciones, donde sus formas y modalidades cambian progresivamente por el mismo efecto de dichas interacciones" (Muller y Surel, 1998:54). Esta perspectiva integra dos dimensiones al análisis del accionar público: "las instituciones como factor de orden" y al mismo tiempo "como interpretaciones del mundo" (Muller y Surel, 1998). Las instituciones van así a determinar las reglas del juego y de esta manera intervenir no sólo en la acción de los gobiernos sino también en la manera como los actores implicados deciden conducir sus acciones.

El enfoque tridimensional presentado aquí no se inscribe en una perspectiva lineal como aquella descrita por el ciclo de Jones (1970); al contrario, va más allá de las fases de construcción de un problema, de agenda, decisión y evaluación, 
para estar también presente en el proceso de implementación. Se entienden las políticas públicas como procesos integrados en continua reconstrucción. En el sector cultural esta perspectiva es fundamental ya que la relación del hombre con el mundo y con sí mismo está en permanente evolución.

Estas reflexiones preliminares permiten entonces proponer este estudio que se centra en la implementación de una institución pública cuyo análisis no escapa de la intervención de un proceso global que se lleva a cabo a nivel estatal: la modernización y reforma del estado emprendida desde el regreso de la democracia en 1990, que generará tensiones particulares al interior de la institución, provocando "efectos inesperados" y dinámicas, algunas, contradictorias con el proyecto original basado en el paradigma cultural representado por el lema "la cultura es tarea de todos".

\section{LAS TENSIONES DE LA IMPLEMENTACIÓN Y LA INDEFINICIÓN DE UNA CATEGORÍA DE INTERVENCIÓN PÚBLICA}

Observando el período de implementación de esta nueva institución y más precisamente estudiando la concepción y elaboración del documento fundador de la acción pública en materia cultural, "Chile quiere más cultura: definiciones de política cultural 2005-2010”, se puede analizar la transición desde el diseño de la política a su implementación, lo que lleva a constatar dos dinámicas presentadas en este artículo.

La primera está relacionada a la manera como esta llamada "carta de navegación", que nace desde un espíritu de participación y transparencia, dos pilares del CNCA, se transformará en un "marco rígido" de la acción del estado en este nuevo sector. Para esto se hace necesario abordar el paradigma cultural "la cultura es tarea de todos" que da vida al CNCA.

En segundo lugar es posible observar que esta dinámica correspondiente a la citada necesidad de participación entrará en conflicto con la búsqueda de eficiencia de las organizaciones públicas, el que será potenciado por las exigencias del proceso de modernización del estado en el cual se inscribe esta reforma, originando una "tecnocratización" de la política cultural. 


\section{1 "La cultura es tarea de todos": desde el acto iniciático hacia una política concreta en materia cultural}

Mirar las políticas públicas desde la dimensión de las ideas y más precisamente desde el concepto de paradigma exigió el seguimiento de los hitos que contribuyeron a su cristalización y que tuvieron lugar durante un período de más de diez ańos, donde el debate se centró en el rol que le corresponde jugar al estado en materia cultural. Se trató concretamente del estudio de dos comisiones de discusión sobre la cultura: la Comisión Garretón (donde se propone por primera vez un Consejo Nacional de la Cultura) ${ }^{1}$ y la Comisión Presidencial Ivelic en 1997. Una tercera, de carácter Interministerial, que será organizada por el Presidente Frei en 1998, con el objetivo de elaborar el proyecto de ley de una Institución Cultural para Chile (termina proponiendo una Dirección Cultural y no un Consejo). Este proyecto es ingresado al Congreso en 1998, pero en el 2001, gracias a indicaciones enviadas por el Presidente Ricardo Lagos, se propone finalmente la creación de un Consejo Nacional de la Cultura como fue propuesto por las dos comisiones culturales citadas ${ }^{2}$.

Sin duda la instancia crucial para la esperada creación del CNCA fue el gobierno de Ricardo Lagos, que desde un comienzo mostró un interés por desarrollar este ámbito. El 16 de mayo del 2000 la política cultural es elegida como temática principal de su primer discurso a la Nación. Este discurso pretendía establecer líneas directrices en vista de la celebración del Bicentenario de la Independencia en el año 2010, donde se le atribuyó a la cultura un rol fundamental en el desarrollo del país. Se expresa en dicha ocasión el deseo de construir en el país una política de "estado" y no de "gobierno", es decir, que el desarrollo cultural no quedara condicionado a un programa de gobierno sino que representara una misión que

1 La Comisión Garretón se crea en 1990 por el Ministro de Educación de la época, Ricardo Lagos, y entrega su informe en el año 1991. Dicho informe identifica principalmente los siguientes problemas en el sector cultural: "La dispersión y duplicación de funciones de los diversos ministerios y organismos, la descoordinación entre ellos y el marcado burocratismo, la ausencia de fondos para el desarrollo artístico y cultural, la carencia de una política global de patrimonio cultural y la concentración de los escasos recursos en la capital". Ella va igualmente a destacar la ausencia en el sector público de "un interlocutor institucional para los asuntos de la comunidad artística” (pág. 5)

2 Otra importante instancia en este período fue el «Encuentro de Políticas Públicas, Legislación y Propuestas Culturales». que se llevó a cabo en 1996 en el Congreso Nacional de Valparaíso; gracias a este encuentro fue nombrada la Comisión Presidencial Ivelic. 
va más allá del ritmo impuesto por las elecciones. En términos teóricos se defiende la constitución de una "política cultural" portadora de un proyecto, que en el caso chileno sería contribuir a un desarrollo sustentable.

Se pudieron así constatar las ideas que impulsaron el proyecto cultural, los actores comprometidos y los instrumentos que representaron dichas convicciones. A través de estos elementos se reconoció la cristalización de un paradigma cultural gracias a la identificación de los cuatro elementos que lo constituyen (Kuhn, 2008; Surel, 2005). Los principios generales, las hipótesis y leyes, la metodología y los instrumentos y herramientas específicas. La transcripción de estos elementos al caso chileno se pueden observar en términos teóricos y descriptivos a través del cuadro $\mathrm{n}^{\mathrm{o}} 1$. Se observa que el paradigma cultural funda un modelo: El modelo chileno de política cultural que, en términos generales, transita entre el deber que tiene el Estado de construir los canales apropiados para un óptimo desarrollo cultural, con la responsabilidad que recae en "todos" para apoyar, impulsar y/o ayudar a dicho desarrollo. El estado no debe tener el total de responsabilidades en la materia y, por sobre todo, no le corresponde a él dirigir hacia dónde va la cultura. La sociedad civil, el mundo cultural, la empresa y el mercado son actores indispensables en este modelo, que surge desde los discursos orientados bajo el lema "la cultura es tarea de todos". 


\section{Cuadro No 1}

\section{Síntesis del paradigma cultural cristalizado hacia fines de 1990}

\begin{tabular}{|c|c|c|}
\hline $\begin{array}{l}\text { Elementos que } \\
\text { distinguen un } \\
\text { paradigma }\end{array}$ & Referencia teórica & Caso chileno \\
\hline $\begin{array}{l}\text { Principios } \\
\text { generales }\end{array}$ & $\begin{array}{l}\text { Lo que Kuhn asimila a una } \\
\text { "visión del mundo" que re- } \\
\text { presenta el núcleo cognitivo } \\
\text { de toda actividad científica. }\end{array}$ & $\begin{array}{l}\text { La Cultura: Elemento importante de democratiza- } \\
\text { ción social y vital dentro del contexto global para } \\
\text { la protección de identidades nacionales y locales. } \\
\text { Relación estado/sociedad/cultura: el desarrollo } \\
\text { cultural no es exclusivo del Estado, existen res- } \\
\text { ponsabilidades de la sociedad y del mercado. } \\
\text { Globalización e inserción internacional: una im- } \\
\text { portancia acordada a la industria cultural. } \\
\text { En el 2000: la cultura, eje del desarrollo (período } \\
\text { Lagos). }\end{array}$ \\
\hline $\begin{array}{l}\text { Hipótesis, } \\
\text { leyes o } \\
\text { principios } \\
\text { específicos }\end{array}$ & $\begin{array}{l}\text { Se refiere al conjunto de } \\
\text { "axiomas, hipótesis y razo- } \\
\text { namientos que establecen un } \\
\text { vínculo entre el sistema sim-- } \\
\text { bólico y el universo concreto". } \\
\text { Se trata de normas de acción, } \\
\text { el paso desde el sistema abs- } \\
\text { tracto determinado por el } \\
\text { elemento anterior a un de- } \\
\text { sarrollo lógico que permitirá } \\
\text { la elaboración de normas y/o } \\
\text { líneas de acción. }\end{array}$ & $\begin{array}{l}\text { Necesidad de proteger el sector, y como esta mi- } \\
\text { sión no es exclusiva del estado, hay que desarrollar } \\
\text { iniciativas para que el sector privado invierta en el } \\
\text { sector, así como también generar los canales ne- } \\
\text { cesarios para abrir un espacio a la sociedad civil. } \\
\text { Esto hace necesario desarrollar una expertise de } \\
\text { estado y del mundo privado en la materia. } \\
\text { Legitimación de la acción del estado y de actores } \\
\text { que se encuentran fuera de la esfera pública. }\end{array}$ \\
\hline Metodologías & $\begin{array}{l}\text { Relacionado en políticas pú- } \\
\text { blicas a los procedimientos y } \\
\text { tipos de relaciones estableci- } \\
\text { dos entre el estado y un sec- } \\
\text { tor particular. }\end{array}$ & $\begin{array}{l}\text { Relaciones de fuerza entre el estado y la sociedad } \\
\text { civil, guiados por la mediación y la consultación. } \\
\text { Aparece entonces un espacio de interacción entre } \\
\text { el Foco importante puesto al "experto": estado y } \\
\text { la sociedad basada en comisiones de consultación, } \\
\text { comisiones de "expertos" para tratar las interro- } \\
\text { gantes relacionadas al mundo cultural. El finan- } \\
\text { ciamiento de proyectos artísticos y culturales se } \\
\text { desarrolla a través de una delegación de decisión a } \\
\text { la comunidad artística y cultural. }\end{array}$ \\
\hline $\begin{array}{l}\text { Instrumentos } \\
\text { y herramien- } \\
\text { tas específicas }\end{array}$ & $\begin{array}{l}\text { Dispositivos de intervención } \\
\text { en la sociedad. Los que hacen } \\
\text { realidad el sistema abstracto } \\
\text { definido por los principios } \\
\text { metafísicos generales. }\end{array}$ & $\begin{array}{l}\text { * Ley Valdés (1990). } \\
\text { * Fondos concursables (FONDART en } 1992 \text { y } \\
\text { FNLL 1993). } \\
\text { * Proposición de crear una institución colegiada } \\
\text { (CNCA) que permita la participación de los acto- } \\
\text { res involucrados en el mundo cultural a través de } \\
\text { una institución de tipo Consejo. } \\
\text { Su aplicación más tarde a los Consejos Sub-secto- } \\
\text { riales (música y audiovisual, el Consejo Nacional } \\
\text { del Libro y la lectura ya había sido creado en 1993). } \\
\text { Se pueden considerar más en detalle la ley Valdés } \\
\text { y Fondos concursables. }\end{array}$ \\
\hline
\end{tabular}

Fuente: Muñoz, 2011a 


\subsection{Un ideal cultural nacional: desde lo sońado a lo vivido}

La construcción de la política cultural es impulsada por la función primera que tiene el CNCA de "estudiar, adoptar, poner en ejecución, evaluar y renovar políticas culturales, así como planes y programas del mismo carácter, con el fin de dar cumplimento a su objeto (de apoyar el desarrollo de la cultura y las artes, y de conservar, incrementar y difundir el patrimonio cultural de la nación y de promover la participación de las personas en la vida cultural del país)" (Ley No 19.891, 2003). A partir de esta perspectiva el CNCA aspira en su primer año de existencia a implementar un esquema institucional participativo con el fin de determinar líneas de acción para el período 2005-2010, que además fundarían la política cultural. Se elabora así una "carta de navegación", titulada "Chile quiere más cultura, definiciones de política cultural 2005-2010”, publicada junto al diario La Nación en el mes de mayo de 2005. Este documento resultó de un trabajo coordinado por el Directorio del CNCA y contó, gracias a la Convención Cultural del 2004, con la participación de los representantes del mundo cultural a nivel nacional a través de los Consejos Regionales y Consultivos ${ }^{3}$.

Esta primera Convención de la Cultura representó un acto histórico y/o iniciático por dos razones, primero porque la creación del CNCA representaba la conquista de un sueño de "institucionalización cultural" esperada ya desde los años '50, cuando José Balmes, acompañado de un grupo de artistas, solicitaron al Presidente de la República de ese entonces, Carlos Ibáńez del Campo, la creación de un Ministerio de la Cultura. En segundo lugar, esta primera Convención representaba en sí la primera vez que se organiza en el país una reunión de tal envergadura para tratar temas en materia de cultura. Estos dos factores marcan profundamente el debate y cargan la correspondiente política cultural de un carácter idealista. La creación de la carta de navegación constata la transcripción del paradigma cultural en la construcción de una política concreta en materia cultural que defiende "el establecimiento de una nueva relación entre el estado, la sociedad y la cultura” (CNCA, 2005: 5). Siguiendo los elementos que conforman el paradigma se puede decir que la Convención se convirtió en el lugar donde se catalogaron por primera vez -a tal nivel- las demandas

3 Se proponen en esta carta 52 medidas que surgen de cinco ejes definidos y observables en el cuadro no 2 (la Creación Artística y Cultural, la Producción Artística y Cultural e Industrias Culturales, la Participación en la Cultura: Difusión, el Acceso y Formación de Audiencias, el Patrimonio, Identidad y Diversidad e Institucionalidad Cultural). Estas medidas pretenden transformarse en nuevas leyes, programas, fondos, infraestructuras culturales y nuevas instituciones. 
del mundo cultural, esto respetando el valor dado a la cultura como factor de democratización social y respetando también el principio de participación en el origen del proyecto (principios metafísicos generales), a través de los cuales nace la postura "la cultura es tarea de todos", (hipótesis) que construyó el "mundo cultural" en ese momento, estableciendo también relaciones basadas en los consensos (metodologías).

Sin embargo será principalmente a partir de la llegada de Paulina Urrutia, la segunda Ministra de Cultura, en marzo 2006, que se identifica el nacimiento de dos tensiones donde se reconocen las primeras contradicciones entre diseño e implementación de una política pública. Esto cuando se implementa dicha carta a través de una solicitud a cada departamento pertinente de trascribir los principios $\mathrm{y}$ medidas en acciones concretas.

La primera tensión se relaciona a los niveles implicados en la toma de decisiones: por ejemplo si se observan los ejes estratégicos y las medidas correspondientes a su cumplimiento (cuadro no2), surge la pregunta: ¿¿de qué manera darle cuerpo a uno u otro eje?, si no es a través de la implementación de una serie de programas e instrumentos que deben ser por ley tratados y definidos por el Consejo (órgano Directivo del CNCA) y no por un departamento preciso (como fue solicitado en dicha ocasión). Además, en este caso la ejecución de esta medida no depende sólo del CNCA, ya que el ámbito del patrimonio compete también a otras reparticiones públicas (Dirección de Bibliotecas, Archivos y Museos DIBAM / Consejo de Monumentos Nacionales -CMN) con las cuales hasta el día de hoy el Consejo se encuentra en una relación de "competencia", más que de la pretendida coordinación enunciada por la ley que crea el CNCA.

De esta manera, al referirse a los niveles de decisión implicados en una política es importante destacar que en el ámbito cultural se complejiza dicha reflexión, ya que se trata de un sector transversal e institucionalmente disperso. Además de subrayar el hecho de que el período de implementación representa en sí mismo nuevas batallas de redefiniciones y/o redecisiones importantes de considerar al momento de estudiar las articulaciones entre lo "pensado" o "sońado" y lo "vivido" de una política pública, en otras palabras, entre los ciclos de decisión/diseño y aquel de la implementación.

La segunda tensión surge en el seno de la organización, que comienza a ser ritmada por el cumplimiento de los objetivos preestablecidos, situación que conduce a una dinámica de lograr "a toda costa" esta transcripción solicitada por la Presidenta- Ministra 
(y posterior evaluación). La organización comienza a ser "gobernada por las reglas del procedimiento" (Crozier y Friedberg, 2007), y surgen interrogantes respecto a esta lucha entre la buena eficiencia que califica positivamente el trabajo de una institución pública con el encierro de la organización dentro de reglas y obligaciones que al mismo tiempo le impiden cumplir con los otros objetivos previstos. Un fenómeno particular, considerando que la traducción de principios en acciones concretas pretendía justamente dar respuesta a los objetivos de la política cultural y a las funciones del Consejo. La implementación de la política cultural se encuentra tensionada entonces entre una declaración de intenciones, representada por el citado documento, y la implementación de la política en la realidad; se pone en marcha una lucha entre el deseo de participación y transparencia con la necesidad de eficacia que el proyecto porta. Se observa que una lógica a largo plazo, necesaria al desarrollo cultural, se ve obstruida por el corto plazo que comienza a ser gobernado por dinámicas que, en este caso -y aun cuando son necesarias-, ponen en riesgo el logro de objetivos.

Se puede recalcar aquí que "los factores que se encuentran en el origen de una institución pueden no ser los mismos que aquellos que la reproducen en el tiempo" (Stinchcombe, 1968, en Thelen, 2003:14); como consecuencia se puede observar una desviación de los resultados esperados y la presencia de efectos adversos que se interponen a la consagración de la misión inicial de las políticas. En esta perspectiva es importante recordar entonces la reflexión sobre la dificultad en identificar e interpretar los problemas y sobre el hecho de que "las políticas públicas no sirven para solucionar los problemas" (Muller y Surel, 1998:31); sin querer negar la existencia y la posibilidad de resolver un problema (necesario al accionar público), estos autores subrayan a través de esta afirmación el hecho de que dicha dinámica es bastante más compleja y que estudiar las políticas públicas como espacios de creación de sentido implica entender, por ejemplo, que resolver un problema conlleva la construcción de "representaciones de problemas", donde interviene el contexto sociopolítico y la estructura misma del estado.

Es, en efecto, dentro de la estructura misma del estado donde se encuentran ciertas características que van a actuar como un referencial global. Se abordarán concretamente las dinámicas impulsadas por el proceso de modernización y reforma del estado, que no sólo están relacionadas con la citada rigidez en la cual podrían encerrar a la organización sino que también influyen fuertemente -como se verá a continuación- en los mecanismos de legitimación de los diferentes actores. 


\section{Cuadro No 2 \\ Síntesis de la Carta de Navegación}

«Chile quiere más cultura, Definiciones de política cultural 2005-2010».

Principios, líneas estratégicas y medidas.

- Afirmación de la identidad y la diversidad cultural de Chile

- Libertad de creación y expresión

- Participación democrática y autónoma de la ciudadanía en el desarrollo cultural

- Rol insustituible y deber del estado

- Educar para la apreciación de la cultura y la formación del espíritu reflexivo y crítico

- Preservación, conservación, difusión del patrimonio cultural y rescate de la memoria

- Igualdad de acceso al arte, los bienes culturales y las tecnologías

- Descentralización de la política cultural y desarrollo cultural equilibrado

- Profundizar la inserción en el mundo

Creación Artística y 1-10: Apoyar al artista en la creación de sus obras, desarrollando

Cultural instancias para una formación de excelencia, facilitando los medios para producir y difundir sus trabajos y mejorando las condiciones para una adecuada inserción en la sociedad.

Producción Artística y $11-17$ : Promover el desarrollo de una industria cultural que aporte Cultural e Industrias al crecimiento de la oferta de bienes y servicios culturales en el país, Culturales y que asegure la difusión de la creación artística y del patrimonio nacional.

Participación en la Cultura: Difusión, Acceso y Formación de Audiencias

18-21: Crear y desarrollar más y mejores audiencias difundiendo la cultura, aumentando la infraestructura, estimulando la gestión, ampliando la formación para la apreciación de las artes e instando por una mayor calidad de los medios de comunicación.

22-26: Aumentar el acceso de los grupos de escasos recursos y de los grupos vulnerables a los bienes de consumo cultural, generando las condiciones iniciales para una relación permanente entre los miembros de estos grupos y la actividad cultural.

27-29: Fomentar la participación y la organización ciudadana descentralizada con fines culturales.

Patrimonio, Identidad y Diversidad

Institucionalidad Cultural
30-39: Preservar, enriquecer y difundir el patrimonio cultural del país, aumentando la inversión e implementando modernas y creativas formas de participación por parte de la comunidad.

40-42: Reconocer y proteger la diversidad cultural de Chile, potenciando la participación cultural de los distintos grupos que conforman la nación y fomentando la expresión de sus prácticas culturales.

43-46: Promover la riqueza del lenguaje y la lectura, generando hábitos y mejorando el acceso al libro.
47-50: Consolidar la nueva institucionalidad cultural en lo relativo a atribuciones, infraestructura, recursos humanos, organizacionales y financieros.

51-52: Estimular el aporte del sector privado a la cultura, perfeccionando los mecanismos tributarios y promoviendo su participación en la gestión cultural.

Fuente: Chile Quiere más Cultura (CNCA, 2005). 


\subsection{La profesionalización de la cultura y la importancia de la experticia: la "tribu" frente a los "tecnócratas"}

La constitución de una profesionalización se puede observar a partir del concepto de expertise que aparece frente a la ausencia de conocimientos en materia cultural a comienzos de la década de los noventa. El período de modernización y reforma del estado imprimirá una dirección centrada en la eficiencia en un sector difícil de delimitar y caracterizado por su carga simbólica que tendrá consecuencias importantes en la recomposición de los campos de poder.

Esta noción de expertise se relaciona a dos perspectivas fundamentales al momento de interiorizarse y estudiar la acción pública: la primera de ella es aquella que pone el acento en "el rol que la experticia debería (o no debería) tener para mejorar la acción pública" (Knowledge in); la segunda se abocará fundamentalmente a "describir cómo las ideas, normas y/o teorías defendidas por los expertos afectan la producción de políticas públicas" (Knowledge off) (Saint y Martin, 2004: 209). En este artículo este concepto se aborda a través de sus dos dimensiones, esta perspectiva integradora es indispensable ya que la expertise es en términos generales «el encuentro entre una coyuntura problemática y un saber especializado» (Restier y Melleray, 1990:547).

Este concepto que modela el paradigma cultural a través de los objetivos de eficiencia y eficacia, e interviene en el aprendizaje de los diferentes actores implicados durante todo el proceso de institucionalización de la cultura, será también fuente de legitimidad para actores específicos, interviniendo en las modalidades de la participación legitimando un nuevo actor, el "gestor cultural". Es decir, se transforma en un elemento importante al momento de estudiar la construcción de legitimidad institucional y actoral en la esfera pública y privada. Es entonces a partir de estas reflexiones que el concepto de expertise se asocia aquí a la competencia individual o colectiva, tanto como a "la situación (o procedimiento) en la cual son llamados a intervenir los expertos" (Restier y Melleray, 1990). Desde esta perspectiva se podrá observar cómo la "expertise" se convertirá en fuente de tensión entre dos prácticas distintas al interior de la organización: entre el proceso de tecnocratización de las políticas culturales identificable en la implementación del CNCA y la herencia de la División Cultura representada por la "tribu Di Girólamo", inmersa en una práctica participativa a través del concepto de ciudadanía cultural. 


\section{CONFLICTO GLOBAL/SECTORIAL: LA AGENDA DE MODERNIZACIÓN Y DE REFORMA DEL ESTADO, UN MECANISMO DE LEGITIMACIÓN DE LOS "EXPERTOS"}

Los procesos de modernización y reforma del estado se presentan como una oportunidad de entrar en la realidad democrática del país y más precisamente en el tema que interesa aquí, la construcción de una categoría de intervención pública, que se traduce por la recomposición de relaciones entre el estado y la Sociedad en un contexto donde era necesario asegurar la reconstrucción de las instituciones democráticas. Con el advenimiento de la democracia este proceso de modernización y reforma se transformó en un imperativo dentro de los gobiernos de la Concertación. La interacción entre este proceso considerado como referencial global, con el referencial sectorial cultural que ha sido tratado bajo el prisma del paradigma cultural, intervendrá en la recomposición de los espacios de poder. La introducción del modelo neoliberal emprendido en Chile durante la década de los setenta y más tarde el contexto histórico de democratización van a impulsar este proceso de reforma y modernización que intervendrá en el conjunto de la administración pública.

Los períodos de modernización y reforma emprendidos durante la década de los ochenta en la región Latinoamericana implicaron una serie de reformas "asociadas a una reducción del rol del estado como productor directo, una transferencia de protagonismo al sector privado y un fortalecimiento del rol regulador del estado en el marco de las nuevas realidades vinculadas al progreso tecnológico y la globalización" (Stark, 2001:1). Reformas orientadas hacia la constitución de un estado regulador que, más que remplazar al mercado cuando este se mostraba defectuoso, pretendía acompañarlo, apoyarlo y/o complementarlo. Esto implicó la creación de una institucionalización apropiada a estos fines, que incluía la creación institucional si se hacía necesario.

En Chile este proceso de reforma y modernización del estado implicó, durante la década de los noventa, la implementación de una serie de reformas estructurales destinadas a "circunscribir el rol del estado y a reforzar el mercado como principal mecanismo de asignación de recursos en la economía" (SEGPRES, 2006:9). Se replantean así los temas relacionados al rol que le toca jugar al estado en el 
nuevo contexto de reorganización del espacio tanto público como privado. Más tarde los gobiernos y los organismos internacionales focalizan su atención "hacia las instituciones, bajo el predicamento de contar con aparatos públicos fuertes, eficientes, transparentes y creíbles. En una palabra, se busca constituir un estado más orientado al servicio real de los ciudadanos" (SEGPRES, 2006:9).

La evolución del sector público hacia una multiplicación de escalas de acción y de actividades emprendidas para responder a las expectativas de los ciudadanos, cada vez más exigentes, puso en evidencia la necesidad de profundizar los temas de la transparencia, la gestión institucional y el impacto de los recursos empleados.

\section{LA DIVISIÓN CULTURA Y EL CNCA: LA TECNOCRATIZACIÓN DE LA POLÍTICA CULTURAL}

A través del estudio de la implementación se observa que la presencia del paradigma cultural no significa una estabilidad de las ideas fundadoras del CNCA; al contrario, revela que procesos de redefiniciones comienzan nuevamente. En este caso se trata de dos "visiones" y/o "maneras de hacer" al interior de la nueva institución cultural.

La primera surge de la División Cultura y aparece guiada por los valores de participación en la definición de sus líneas de acción, tanto en su personal como en el funcionamiento del departamento. La acción de la División era guiada por el concepto "desde las bases", que refleja la experiencia de los Cabildos Culturales, creados e implementados durante la administración de Claudio di Girólamo, un artista de prestigio a nivel nacional, que dirigió la División desde 1997 hasta el año de creación del CNCA. Los funcionarios públicos de aquel entonces fueron marcados y formados por su gestión, lo que hace proponer el apelativo de "tribu Di Girólamo", que representa el concepto de equipo, donde todos tenían participación dentro de una estructura horizontal ${ }^{4}$. Se trataba en la época de un

4 La coordinadora a nivel nacional de FONDART durante más de diez años afirmará al respecto que se trataba de "una época donde teníamos pocos recursos, pero al mismo tiempo teníamos un equipo humano cohesionado, comprometido y solidario, como lo tienen generalmente las instituciones de estructuras pequeńas" (Nivia Palma, entrevista 2008). 
sector que contaba con poca especialización y donde este grupo aprendió sobre la marcha, la dinámica de "ensayo-error" fue fundamental dentro de su aprendizaje. Mirar la acción del estado tomando en cuenta la noción de aprendizaje permite, en los términos de Hall (1993), tener una mirada de la acción pública que destaca la costumbre de los actores de mirar las experiencias de su pasado, un proceso de ensayo-error donde la acción pública funcionará entonces de acuerdo a ciertas apreciaciones o "pre-juicios" de los caminos existentes5.

Los valores de la división se ven expresados en los Cabildos Culturales que se crean en 1999 teniendo por misión generar un espacio de debate a nivel comunal para consolidar una proposición nacional de desarrollo cultural hacia el Bicentenario de la República en 2010. Promovían el ya citado sistema de participación "desde las bases" buscando construir y promover un verdadero ejercicio de ciudadanía en la elaboración de las políticas públicas. Los Cabildos fueron impulsados por el concepto de "ciudadanía cultural", concepto que surge en Chile gracias a un discurso titulado "Ciudadanía Cultural, una carta de navegación hacia el futuro", pronunciado por Claudio di Girólamo en la "Conferencia Intergubernamental sobre Políticas Culturales para el Desarrollo", en Estocolmo, en el año 1998. En este discurso el Director de la División Cultura se centra en una reflexión sobre la identidad latinoamericana así como también en la importancia de considerar los derechos ciudadanos culturales en las constituciones de la región como una manera de responder a los desafíos impuestos por la globalización. Algunos años más tarde él afirmará respecto a esta ocasión que dicha reflexión se basaba en:

el hecho cierto de que la construcción de una nación, y por ende de su propia identidad, es un proceso siempre inacabado, que necesita del aporte constante y decidido de todos los habitantes de su territorio (...) es un deber del estado el garantizar que cada uno de los sujetos sociales que dan vida a su específica comunidad, pueda entregar, activa y libremente, ese aporte personal en una situación

5 Esta noción valoriza también los mecanismos de "conocimiento" atribuyendo una importancia a los procesos de construcción de experiencia y en consecuencia otorga importancia a los expertos dentro y fuera del aparato político administrativo. Sin olvidar tampoco una autonomía relativa del estado frente a presiones exteriores, delimitando un contexto relativamente estable donde se realiza este aprendizaje. 
de equidad en la que se acojan con la amplitud necesaria y sin trabas de ninguna especie las diferentes propuestas y visiones de mundo (Di Girólamo, 2000:1).

Estos elementos marcan una comprensión de la política cultural "desde el interior", es decir -y en las palabras de Claudio di Girólamo- desde la facultad de cada uno para transformarse en "prosumidores" de cultura. Estos valores construyeron el mundo cultural de aquella época y marcaron al personal de la División, el que actuaba defendiendo dichos valores. Existía en efecto una real consciencia social del impacto de sus acciones sobre los usuarios de las políticas implementadas y se había desarrollado al interior de esta División una identidad incipiente que venía acompañada de prácticas de trabajo participativas y una concepción del rol que ellos se atribuían, basado en un valor social compartido: contribuir al desarrollo cultural del país.

La segunda visión responde al paradigma cultural que funda el CNCA. La cristalización de este paradigma viene acompañada de la legitimación de un nuevo actor: el gestor cultural. Este actor surge de la influencia del management moderno en el mundo de la cultura. La constitución de un sector donde la responsabilidad de "todos" es valorada, la interacción de diferentes esferas y diferentes actores en un marco que privilegia el financiamiento mixto exige un actor capaz de articular, coordinar y activar esta estructura horizontal. Este actor se transforma en una pieza central del panorama cultural chileno, donde es necesaria la construcción de redes, y en la búsqueda de rentabilidad y eficiencia de acciones culturales.

Esta concepción impulsa a los diferentes actores y autoridades políticas a familiarizarse con esta lectura emprendedora de la gestión cultural, ya que un proyecto logrado es un proyecto "bien gestionado", perspectiva además coherente con los objetivos de eficiencia y eficacia impuestos por el proceso de modernización del estado. Se defiende entonces en este contexto una aproximación técnica de proyectos culturales y se insistirá en la presencia de expertos para su realización. Tecnócratas, expertos y gestores se transforman en sinónimos de una acción cultural efectiva.

Esta dinámica que implicaba a diferentes reparticiones públicas, encontraba en el CNCA un nuevo adepto. Él se inscribe en efecto dentro de la línea "ajustes institucionales sectoriales" (SEGPRES, 2006), impulsado bajo el gobierno del 
Presidente Ricardo Lagos y enmarcados dentro de un proceso global de evolución del estado. Es de esta manera que los conceptos de eficiencia, eficacia y expertise se transforman en referentes de la nueva institucionalidad cultural que será administrada por un personal que, en su mayoría, proviene de la División Cultura, portadores como vimos de una concepción del "hacer cultural" distinta. Se producirá entonces un choque entre estas dos prácticas intelectuales, entre la organización abierta, participativa y horizontal de la División con aquella que se implementa a través del CNCA, marcada por una tecnocratización de las políticas culturales. Una confrontación entre la "tribu Di Girólamo" y los "tecnócratas", representantes de la gestión y la eficacia.

Esta tensión dificulta la implementación y esta situación se puede comprender por el hecho de que el sector cultural, así como también el del Patrimonio, son sectores sensibles a los cambios y donde dichos cambios no pueden realizarse en ausencia de sus "implementadores". Estudios en el sector del patrimonio subrayaron la importancia de incorporar los equipos humanos en los procesos de cambio institucional. La conferencia de Nara realizada en 1994, ya ponía el acento en "los aspectos inmateriales o "valores asociativos" que pueden completar la evaluación y la conservación del patrimonio material” (Matsuura, 2002). Este tema no se puede descuidar, más aún cuando se trata de sectores de intervención cargados de valores simbólicos que conducen a los actores a subjetivar sus roles, elemento fundamental en el funcionamiento de las políticas públicas y especialmente en el sector cultural. Un aspecto que la racionalización de la gestión pública no considera y que produce tensiones al interior del CNCA.

A esta instalación progresiva de creencias fuertemente ligadas a prácticas tecnócratas entre los años 2003 y 2008, se suma un costo adicional a la organización que parece no haber sido considerado ni evaluado: la mudanza a Valparaíso. Esta situación contribuyó a la fuga de funcionarios de la División Cultura y formados en la "tribu Di Girólamo"; se trataba de las únicas personas que tenían una experiencia en la gestión pública de la cultura y, sobre todo, se habían constituido en los interlocutores del mundo artístico. De esta manera se perdía un "savoir faire" que no pudo ser considerado, integrado o evaluado para el buen funcionamiento de la nueva institución. 
Esta situación confirma lo que afirman Jobert y Muller (1987) en referencia a las organizaciones, cuando destacan que "las administraciones no son sólo instrumentos entre las manos de gobernantes. Ellas están formadas por grupos humanos que intentarán valorizar su propia situación a través de la acción pública” (Jobert y Muller, 1987:42). Se puede también constatar que los programas fueron percibidos durante la etapa estudiada como "imperativos sancionados" (Lascoumes, Le Galès, 2007: 41) y desde ahí constituyen difícilmente recursos para la acción. Las perspectivas centradas en los resultados (que permiten medir la eficiencia y eficacia) tienen consecuencias en la acción pública al impedir una mirada global de conjunto y, aún más, orienta la organización a cumplir con las demandas y no a integrar, ser parte o "sentirse" parte de un proyecto a largo plazo, elementos fundamentales para el desarrollo del sector cultural.

Un informe sobre la experiencia chilena del sistema de control de gestión y presupuestos por resultados afirma en efecto que "El creciente énfasis en el desarrollo de información de desempeño, en la forma de mediciones y/o evaluaciones, ha tenido por objeto cambiar el foco desde los insumos a los resultados de las acciones públicas" (DIPRES, 2005:14). Las problemáticas relacionadas a la expertise y fomentadas por el marco ya descrito se transforman en uno de los elementos que marcan el ritmo de la implementación de la nueva institución y harán al mismo tiempo resurgir los debates sobre el rol del estado en materia cultural, principalmente el debate nacido por la proposición ya presente, a media voz, en el informe Garretón de 1990: la necesidad de crear un Ministerio de la Cultura.

Los temas sobre el tipo institucional pone problemas a la acción del Consejo, ya que en el marco existente dicho Consejo pierde autonomía frente al Ministerio de Educación: alguno de sus servicios están aún en dependencia de dicho ministerio. Esto dificulta también la evaluación de las políticas desarrolladas por el CNCA, quien es mirado y sobre todo juzgado como un Ministerio cuando en la realidad institucional y organizacional no lo es. Un ejemplo de este caso se puede observar gracias a una evaluación de FONDART, que estudia el período 20042007, realizada por la Dirección de Presupuesto (DIPRES) publicada en 2008. Este informe subraya principalmente la ausencia de diagnósticos, de indicadores y de seguimientos que impide medir el impacto de este instrumento en el desarrollo cultural al cual él pretende contribuir. La respuesta de la entonces Ministra Presidenta del CNCA, Paulina Urrutia, fue que era necesario "contextualizar 
adecuadamente el Programa dentro del marco institucional en el que se inscribe, el cual no constituye una organización pública tradicional, la de un servicio público estructurado como Ministerio, sino que asume la naturaleza de un Consejo Nacional" (DIPRES, 2008: 3). Aparecen entonces ciertas ambigüedades respecto a las miradas que existen hacia esta nueva institución que fue presentada como "innovadora" a la tradición institucional chilena ${ }^{6}$ y que sigue siendo percibida o entendida como Ministerio.

Esta figura "híbrida" como fue calificada por algunos parlamentarios durante los años del debate en el Congreso Nacional, se convierte en el flanco de reinterpretaciones y entrecruzamientos de responsabilidades, de intervenciones y de relaciones de fuerza por los propios actores en juego. Un conflicto adicional que vendrá, de cierta manera, a encerrar al "Presidente Ministro" en la ambigüedad permanente de ser un Ministro sin Ministerio.

\section{Corolario: Hacia una tipología del experto}

La tecnocracia se ha convertido en un tema de múltiples investigaciones orientadas principalmente a estudios guiados por la sociología de las élites. Se pretende comprender el efecto de la importancia progresiva que los expertos han adquirido al interior de la administración pública. La dupla tecnócrata y político motivan debates a fin de decidir cual de estos grandes actores aparecen los más aptos a ocupar altos cargos en la administración pública. La valorización de los mecanismos de conocimiento producida por el proceso de modernización y reforma del estado ejerce, en este tema, una influencia fundamental. El paradigma cultural de nivel sectorial y el proceso de reforma y modernización del Estado a nivel global contribuyeron paralelamente a consagrar la expertise como eje principal de la política cultural, lo cual entregó a los expertos un lugar preponderante.

Se busca combinar los análisis orientados desde una perspectiva clásica de las élites -guiada por un análisis vertical y centrado en la toma de decisiones/construcción de legitimidad política- con otra entrada que considera los mecanismos de legiti-

6 Aun cuando este tipo institucional responde más bien a una continuidad respecto a las instituciones históricamente creadas en materia cultural, como también al tipo institucional implementado por la Concertación (SERNAM, CONAMA, CNTV entre los principales). 
mación para comprender sus efectos en los “implementadores" de las políticas y, desde esta perspectiva, en las legitimaciones profesionales. Según esta perspectiva se instala entonces la pregunta: ¿quiénes son hoy los expertos que el estado necesita? Y para intentar responder esta pregunta se propusieron en un primer tiempo ciertos elementos que permitieron constatar el tipo de expertise legitimada en el momento de creación del CNCA. Se trató de una combinación entre el rol que esta expertise tuvo en dicho momento y el reconocimiento de las ideas defendidas por los expertos en cuestión. Se pudo observar que si bien el paradigma cultural defendía la necesidad de una expertise, es el proceso de modernización que va a redireccionar esta necesidad hacia el reconocimiento de un experto: el tecnócrata. Una situación que condujo a una no valoración de prácticas y de un savoir faire de otro tipo de actores, como por ejemplo los administradores culturales de la División Cultura.

Se propone en un segundo momento una tipología general del experto que pretende entrar en el concepto de "tecnócrata" para comprenderlo desde diferentes aspectos que contribuyen a constituir la capacidad de un actor a acceder a "recursos" y/o "repertorios de acción" que construyen su legitimidad y su capacidad de intervenir en los procesos de políticas públicas.

Primero se encuentra el experto relacionado a la "profesionalización", al savoir faire profesional del sector cultural, que reconoce al "gestor cultural"; como ya se expuso el gestor surge de la necesidad de contar con un actor que sea capaz de articular, coordinar y poner en marcha esta estructura diversa y de carácter transversal defendida por el paradigma cultural. Este actor será reconocido formalmente en el Informe de la Comisión Ivelic (1997: 47), que en sus anexos lo presenta como aquel capaz de "orquestar" los diferentes actores del mundo cultural.

7 Se entiende aquí por recursos "las características que dotan a los actores de una capacidad de acción o más precisamente les aseguran cuotas de poder" (Muller y Surel, 1998:81), y que pueden provenir de la posición que ocupen, de las ideas que defiendan y/o de los grupos a los cuales pertenezcan o se asocien. Los repertorios de acción son por su parte entendidos como "el conjunto de medios puestos en marcha para ejercer poder, generalmente bajo la forma de una influencia sobre los que toman la decisión en la esfera pública, bajo la fase de agenda de problemas sociales como de implementación de políticas públicas" (Muller y Surel, 1998:83). Se trata, por ejemplo, de las diferentes formas de manifestaciones, ya sean pacíficas u organizadas con algún objetivo, como por ejemplo las huelgas, movilizaciones, reuniones colectivas, entre otras. 
El segundo tipo de experto es el intelectual que participa igualmente como consejero de gobierno, quedando al mismo tiempo inserto en la investigación y la academia. No se considera a sí mismo como un tecnócrata pero suele intervenir en la conducción de las políticas públicas de la misma manera. Su influencia está asociada a las condiciones sociales, históricas y políticas de transición y consolidación democráticas y a las necesidades que este contexto exigió para la creación e implementación de un nuevo modelo de desarrollo. En este contexto se posiciona más en el referencial global y no sectorial como el gestor cultural. Él permanece próximo al poder pero no "en" el poder; su flexibilidad y autonomía se ven reducidas respecto al experto profesional que puede recorrer las diferentes esferas con el fin de integrarlas. Este "intelectual" está más relacionado a un gobierno o a una coalición política, lo cual reduce su campo de acción.

El tercer tipo de experto es el tecnócrata que hace referencia aquí a un experto que puede ser tanto consejero de estado como miembro del gobierno. Él está, de esta manera, más "politizado" que el tipo de experto descrito anteriormente, ya que participa desde "dentro" en la acción pública. Esta situación le da menos flexibilidad y autonomía ya que será reconocido o asociado a un gobierno particular y a un campo particular de expertise.

Estos tres tipos de actores, que definen también tres maneras de actuar y diferentes tipos de flexibilidad a transitar entre las esferas y niveles de acción, un factor fundamental para la constitución de la autonomía de un actor frente a presiones exteriores.

Este estudio, que se centró en la construcción de una categoría de intervención pública, permitió mostrar dinámicas que contribuyen a una reflexión sobre las herramientas con las que se cuenta hoy para estudiar las políticas públicas. Las características propias del sector cultural revelaron de manera más evidente cómo, para emprender un estudio sobre la implementación de una institución sectorial, es necesario integrar el contexto global de evolución del estado. Esta particularidad instala un elemento a considerar, donde la evolución de las estructuras administrativas y las dificultades para construir instrumentos que permitan medir de mejor manera los resultados de un sector van a instalar dinámicas que ponen en riesgo los objetivos iniciales de la política misma. Es importante destacar respecto a este tema que no se pueden considerar las políticas públicas como algo "dado", 
es decir, donde por ejemplo, la sola creación de una institución aseguraría la obtención de los objetivos para los cuales dicha institución fue concebida. El proceso de políticas públicas expuesto desde una mirada tridimensional muestra que pueden en efecto surgir tensiones contradictorias con los proyectos iniciales y que reconsiderar el estudio de la puesta en marcha permite entrar en las redefiniciones presentes en la acción pública.

Se pudo constatar igualmente cómo las ideas que constituyen el paradigma "la cultura es tarea de todos" defienden un ideal cultural nacional de participación y desarrollo igualitario así como también la necesidad de contar con expertos en materia cultural. Este último elemento, fuertemente intervenido por el contexto global de modernización y reforma, producirá la constitución de dinámicas elitistas producto de la tecnocratización de las políticas culturales. En otras palabras, los cambios de paradigma, o mejor dicho, las tensiones entre paradigmas, van a constituir el CNCA como ejemplo a través del cual se hacen más entendibles temas recurrentes como la tecnocratización de las políticas públicas y la legitimación del actuar público.

Por otra parte la existencia de un paradigma evaluador que imprime a la política pública mecanismos de sanción puede comprometer la vivencia de la misma y provocar su incapacidad para cumplir con el fin para el cual fue creada. Se revela aquí la importancia de reflexionar sobre instrumentos de gestión y control de un sector marcado por lo "abstracto", donde poner cifras o indicadores a intervenciones artísticas y/o culturales es una tarea compleja y por sobre todo donde los efectos de las políticas públicas, de largo plazo, se oponen a las cadencias del politics marcadas por el ritmo electoral. Aquí es donde recobra toda su fuerza el ideal cultural expresado por las autoridades públicas cuando afirmaban la necesidad de construir una política de estado y no de gobierno.

Se puede entonces constatar el interés de comprender las políticas públicas de una manera integradora, no lineal sino en constante evolución, como un espacio donde se construyen y se conjugan las relaciones tanto de la sociedad en su conjunto como referencial global, como de cada sector y cada grupo de actores que la conforman como referencial sectorial. 
Este análisis propuso que las instituciones son el reflejo de las sociedades en las cuales están inmersas y que se encuentran en un perpetuo proceso de reconstrucción, autolegitimación y reconfiguración por los propios actores. Al ser "ideas en acción”, es necesario comprender los mecanismos a través de los cuales se llegan a imponer los "marcos cognitivos y normativos" (Muller, 2004b). En otras palabras, las políticas públicas cristalizan las ideas que rigen un país, es por esto que al estudiar el "vivir" mismo de dichas políticas, es posible abarcar amplios espectros de conflictividad y tensiones que atraviesan las sociedades modernas.

\section{REFERENCIAS BIBLIOGRÁFICAS}

Boussaguet, Laurie; Jacquot, Sophie; Ravinet, Pauline (Dir). (2004). Dictionnaire des Politiques Publiques. Francia: Presses de Sciences Po.

Calmettes, Joël; Djian, Jean-Michel (2003). Culture, une Affaire d'État. La Cinquième République des Affaires Culturelles [DVD]. Francia: Morgan Production; France 5; INA.

Comisión Asesora de Cultura (1991). Propuesta para la Institucionalidad Cultural Chilena. Comisión Consejera en Cultura. Chile: Ministerio de Educación.

Comisión Asesora Presidencial en Materias Artístico-Culturales (1997). Chile Está en Deuda con la Cultura. Chile: Ministerio de Educación.

Consejo Nacional de la Cultura y las Artes (2005). Chile quiere más Cultura. Definiciones de Política Cultural: 2005-2010. Chile: CNCA. Disponible en: http://www.sicsur.org/archivos/documentos/Chile_2005_2010.pdf

Crozier, Michel; Friedberg, Erhard (2007). L'acteur et le Système. Francia: Éditions du Seuil.

Di Girolamo, Claudio (2000). Ciudadanía cultural; construcción del futuro. Documento no publicado, entregado en entrevista realizada en el año 2008. Santiago, Chile.

Dirección de Presupuestos (2005). Sistema de Control de Gestión y Presupuestos por resultados; la experiencia chilena. Chile: Ministerio de Hacienda. Disponible en: http://www.dipres.cl/572/ propertyvalue-15409.html

Dirección de Presupuestos (2008). Informe Final de Evaluación del Fondo Nacional de Desarrollo Cultural, 2004-2007. Chile: Ministerio de Hacienda.

Dubois, Vincent (1999). La Politique Culturelle: Genèse d'une Catégorie d'Intervention Publique. Francia: Belin. 
Hall, Peter (1993). "Policy Paradigm; Social Learning and the state". Comparative Politics Vol. 25, No 3, pp. 275-296.

Hall, Peter; Taylor, Rosemary (1997). "La science politique et les trois néo-institutionnalismes". Revue française de science politique Vol. 47, No3, pp. 469-496.

Jobert, Bruno; Muller, Pierre (1987). L’État en Action: Politiques Publiques et Corporatismes. Francia: Presses Universitaires de France.

Jones, Charles (1970). An Introduction to the Study of Public Policy. Belmont: Duxbury Press.

Kuhn, Thomas (2008). La structure des révolutions scientifiques. Francia: Flammarion; Champs Sciences. Lascoumes, Pierre; Le Galès, Patrick (2007). Sociologie de l'Action Publique. Francia: Armand Colin.

Matsuura, Kö̈chiro (2002). La sauvegarde du patrimoine culturel matériel et immatériel : vers une approche intégrée. Disponible en: unesdoc.unesco.org/images/0013/001372/137245f.pdf

Meny, Yves; Thoening, Jean-Claude (1992). Las politicas Públicas. España: Ariel.

Muller, Pierre; Surel, Yves (1998). L'Analyse des Politiques Publiques. Francia: Montchrestien.

Muller, Pierre (1995). "Les Politiques Publiques comme construction d'un rapport au monde". En Faure A., Pollet G. \& Ph. Warin (dir). La Construction du sens dans les politiques publiques: Débats autour de la notion de référentiel. Francia: L'Harmattan, pp. 153-179.

Muller, Pierre (2003). Les Politiques Publiques. Francia: Presses Universitaires de France.

Muller, Pierre (2004a). "L'État en action revisité". Pôle Sud Revue de science politique de l'Europe méridionale. No 21 , pp. 33-42.

Muller, Pierre (2004b). "Secteur”. En Boussaguet Laurie; Jacquot Sophie; Ravinet Pauline (Dir). Dictionnaire des Politiques Publiques. Francia: Presses de Sciences Po, pp. 405-412.

ICOMOS. Conseil International des Monuments et des Sites (1994). Document Nara sur l'Authenticité. Disponible en: http://www.international.icomos.org/charters/nara_f.htm

Muñoz, Norma (2011a). L'État et la culture au Chili; 1989-2008: les enjeux et défis d'un processus de construction d'une catégorie d'intervention publique. Tesis de Doctorado. Francia: Université Sorbonne Nouvelle; Paris III; IHEAL.

Muñoz, Norma (2011b). "La Culture au Chili: réflexions sur un processus de constitution d'une catégorie d'intervention publique". Cahiers des Amériques Latines; 67. Francia: La Documentation Française.

North Douglas (1990). Institutions, Institutional Change and Economic Performance. New York: Cambridge University Press. 
Palier, Bruno; Surel, Yves (2005). "Les «trois I» et l'analyse de l'État en action”. Revue française de science politique Vol. 55, No1, pp. 7-32.

Poirrier, Philippe (1998). Histoire des Politiques Culturelles de la France Contemporaine. Francia: Bibliest.

Restier-Melleray, Christiane (1990). "Experts et expertise scientifique. Le cas de la France”. Revue Française de Science Politique Vol. 40, No 4, pp. 546-585.

Saez, Guy (1985). "Les politiques de la cultura”. En Leca Jean; Grawits Madelaine. Traité de Sciences Politiques IV. Francia: Presses Universitaires de France, pp. 387-442

Saint-Martin, Denis (2004). "Expertise”. En Boussaguet Laurie; Jacquot Sophie; Ravinet Pauline (Dir). Dictionnaire des Politiques Publiques. Francia: Presses de Sciences Po, pp. 209-217.

Secretaría General de la Presidencia (2006). Reforma del Estado en Chile 1990-2006. Chile: SEGPRES.

Stark, Carlos (2001). Regulación; agencias reguladoras e innovación de la gestión pública en América Latina. CLAD: Naciones Unidas. Disponible en: http://unpan1.un.org/intradoc/groups/ public/documents/clad/clad004

Surel, Yves (1995). "Les politiques publiques comme paradigmes". En La Construction du sens dans les politiques publiques; débats autour de la notion de référentiel. Francia: L'Harmattan, pp. 125-151.

Surel, Yves (1997). L'État et le livre. Les politiques publiques du livre en France (1957-1993). Francia: L'Harmattan.

Thelen, Kathleen (2003). "Comment les Institutions évoluent: perspectives de l'Analyse comparative Historique”. L’Année de la Régulation No 7; pp. 13-43.

Urfalino, Philippe (1989). "Les Politiques Culturelles: Mécénat Caché et Académies Invisibles". Revue L'Année Sociologique No 39; pp. 81-109.

Urfalino, Philippe (2004). L'Invention de la Politique Culturelle. Francia: Hachette.

Recibido: 25 mayo 2011 / Aprobado: 15 noviembre 2011 